\title{
Does Quantum Mechanics Need Interpretation?
}

\author{
Louis Marchildon \\ Département de physique, Université du Québec, \\ Trois-Rivières, Qc. Canada G9A 5H7 \\ email: marchild@uqtr.ca
}

\begin{abstract}
Since the beginning, quantum mechanics has raised major foundational and interpretative problems. Foundational research has been an important factor in the development of quantum cryptography, quantum information theory and, perhaps one day, practical quantum computers. Many believe that, in turn, quantum information theory has bearing on foundational research. This is largely related to the so-called epistemic view of quantum states, which maintains that the state vector represents information on a system and has led to the suggestion that quantum theory needs no interpretation. I will argue that this and related approaches fail to take into consideration two different explanatory functions of quantum mechanics, namely that of accounting for classically unexplainable correlations between classical phenomena and that of explaining the microscopic structure of classical objects. If interpreting quantum mechanics means answering the question, "How can the world be for quantum mechanics to be true?", there seems to be no way around it.
\end{abstract}

\section{Introduction}

Ever since it was proposed more than 80 years ago, quantum mechanics has raised great challenges both in foundations and in applications. The latter have been developed at a very rapid pace, opening up new vistas in most branches of physics as well as in much of chemistry and engineering. Substantial progress and important discoveries have also been made in foundations, 
though at a much slower rate. The measurement problem, long-distance correlations, and the meaning of the state vector are three of the foundational problems on which there has been and still is lively debate.

It is fair to say that foundational studies have largely contributed to the burgeoning of quantum information theory, one of the most active areas of development of quantum mechanics in the past 25 years. Quantum information is dependent on entanglement, whose significance was brought to light through the Einstein-Podolsky-Rosen argument 1. The realization that transfer protocols based on quantum entanglement may be absolutely secure has opened new windows in the field of cryptography [2]. And the development of quantum algorithms thought to be exponentially faster than their best classical counterparts has drawn great interest in the construction of quantum computers [3]. These face up extraordinary challenges on the experimental side. But attempts to build them are likely to throw much light on the fundamental process of decoherence and perhaps on the limits of quantum mechanics itself [4, 5].

Along with quantum information theory came also a reemphasis of the view that the wave function (or state vector, or density matrix) properly represents knowledge, or information [6, 7, 8]. This is often called the epistemic view of quantum states. On what the wave function is knowledge of, proponents of the epistemic view do not necessarily agree. The variant most relevant to the present discussion is that rather than referring to objective properties of microscopic objects (such as electrons, photons, etc.), the wave function encapsulates probabilities of results of eventual macroscopic measurements. The Hilbert space formalism of quantum mechanics is taken as complete, and its objects in no need of a realistic interpretation. Additional constructs, like value assignments [9], multiple worlds [10], or Bohmian trajectories [11] are viewed as superfluous at best.

Just like foundational studies have contributed to the development of quantum information theory, many investigators think that the latter can help in solving the foundational and interpretative problems of quantum mechanics. A number of proponents of the epistemic view believe that it considerably attenuates, or even completely solves, the problems of quantum measurement, of long-distance correlations, and of the meaning of the state vector. Their arguments will be briefly summarized in Sec. 2. I will then argue, in Sec. 3, that the epistemic view and related approaches fail to take into consideration that quantum mechanics has two very different explanatory functions, that of accounting for classically unexplainable correlations 
between classical phenomena, and that of explaining the microscopic structure of classical objects. In Sec. 4, I will ask the question of what it means to interpret quantum mechanics, or any scientific theory for that matter. Drawing from the so-called semantic view of theories, I will argue than interpreting quantum mechanics means answering the question, "How can the world be for quantum mechanics to be true?" I will conclude that so construed, an interpretation can hardly be dispensed with 1

\section{The epistemic and related views}

Let us first examine the arguments that advocates of the epistemic view offer to solve the foundational and interpretative problems of quantum mechanics. I should point out that they do not all attribute the same strength and generality to these arguments. Some advocates believe that the problems are completely solved by the epistemic view, while others are of the opinion that they are just attenuated. This distinction, however, is not crucial to our purpose, and I will simply give the arguments as they are typically formulated.

The first problem that is addressed by the epistemic view is the one of the interpretation of the state vector (or state operator, or wave function). As the name suggests, the state vector is normally interpreted as representing the state of quantum systems. It is a matter of debate whether the state it represents pertains to an individual system or to statistical ensembles of systems [15]. But the epistemic view, which goes back at least to writings of Heisenberg [16], claims that it represents neither. It denies that the (in this context utterly misnamed) state vector represents the state of a microscopic system. Rather, it represents knowledge about the probabilities of results of measurements performed in a given context with a macroscopic apparatus, in other words, information about "the potential consequences of our experimental interventions into nature" [8]. This is often set in the framework of a Bayesian approach, where probability is interpreted in a subjective way.

The epistemic view also addresses the notorious measurement problem. Broadly speaking, the problem is the following. Suppose we want to describe, in a completely quantum-mechanical way, the process of measuring a physical quantity $Q$ pertaining to a microscopic system. For simplicity, assume that

\footnotetext{
${ }^{1}$ This paper reformulates the arguments made in [12, 13], and [14, where additional material can be found.
} 
the spectrum of $Q$ is discrete and nondegenerate, and that the normalized eigenvector $\left|q_{i}\right\rangle$ corresponds to the eigenvalue $q_{i}$. The measurement apparatus should then also be considered as a quantum system, which comes to interact with the microscopic system. Let $\left|\alpha_{0}\right\rangle$ denote the initial state of the apparatus. The interaction will represent a faithful measurement of $Q$ if the combined system evolves like

$$
\left|q_{i}\right\rangle\left|\alpha_{0}\right\rangle \rightarrow\left|q_{i}\right\rangle\left|\alpha_{i}\right\rangle
$$

where $\left|\alpha_{i}\right\rangle$ represents a state of the apparatus wherein the pointer shows the value $\alpha_{i}$ (with $\alpha_{i} \neq \alpha_{j}$ if $i \neq j$ ).

If the Schrödinger equation is universally valid, the combined evolution of the microscopic system and macroscopic apparatus is unitary (assuming, unrealistically, that they form together a closed system). But then, an initial state involving the superposition of several eigenstates of an observable of the microscopic system evolves into a final state involving a superposition of macroscopically distinct states of the apparatus (or of the apparatus and environment in more realistic situations). Explicitly,

$$
\left\{\sum_{i} c_{i}\left|q_{i}\right\rangle\right\}\left|\alpha_{0}\right\rangle \rightarrow \sum_{i} c_{i}\left|q_{i}\right\rangle\left|\alpha_{i}\right\rangle .
$$

One solution to this problem appeals to the collapse of the state vector [17, in which the Schrödinger equation breaks down and only one term of the macroscopic superposition (e.g. $\left.\left|q_{j}\right\rangle\left|\alpha_{j}\right\rangle\right)$ remains.

How does the epistemic view deal with the measurement problem? It does so by construing the collapse of the state vector not as a physical process, but as a change of knowledge [18. Insofar as the state vector is interpreted as objectively describing the state of a physical system, its abrupt change in a measurement implies a similar change in the system, which calls for explanation. If, on the other hand, and in line with a Bayesian view, the state vector describes knowledge of conditional probabilities (i.e. probabilities of future macroscopic events conditional on past macroscopic events), then as long as what is conditionalized upon remains the same, the state vector evolves unitarily. It collapses when the knowledge base changes (this is Bayesian updating), thereby simply reflecting the change in the conditions being held fixed in the specification of probabilities.

A third problem which is addressed by the epistemic view is the one of long-distance correlations [8, 19]. Consider the realization of the EinsteinPodolsky-Rosen setup in terms of two spin 1/2 particles (labelled $A$ and $B$ ), 
where the state vector $|\chi\rangle$ of the compound system is an eigenstate of the total spin operator with eigenvalue zero. In this case

$$
|\chi\rangle=\frac{1}{\sqrt{2}}\{|+; \mathbf{n}\rangle \otimes|-; \mathbf{n}\rangle-|-; \mathbf{n}\rangle \otimes|+; \mathbf{n}\rangle\} \text {. }
$$

Here the first vector in a tensor product refers to particle $A$ and the second vector to particle $B$. The vector $|+; \mathbf{n}\rangle$, for instance, stands for an eigenvector of the $\mathbf{n}$-component of the particle's spin operator, with eigenvalue +1 (in units of $\hbar / 2$ ). The unit vector $\mathbf{n}$ can point in any direction, a freedom which corresponds to the rotational symmetry of $|\chi\rangle$.

Suppose Alice measures the $\mathbf{n}$-component of $A$ 's spin and obtains the value +1 . Then she can predict with certainty that if Bob measures the same component of $B$ 's spin, he will obtain the value -1 . If the state vector represents the objective state of a quantum system, it then seems that $B$ 's state changes immediately upon Alice's obtaining her result, and this no matter how far apart $A$ and $B$ are. Since the word "immediately", when referring to spatially separated events, is not a relativistically invariant concept, such a mechanism is not easy to reconcile with the theory of special relativity.

In the epistemic view, what changes when Alice performs a measurement is Alice's knowledge. Bob's knowledge will change either if he himself performs a measurement, or if Alice sends him the result of her measurement by conventional means. Hence no information is transmitted instantaneously, and there is no physical collapse on an equal time or spacelike hypersurface.

Related to the epistemic view is the idea of genuine fortuitousness [20, 21], a radically instrumentalist view of quantum mechanics. The idea "implies that the basic event, a click in a counter, comes without any cause and thus as a discontinuity in spacetime" [21, p. 405]. Indeed

[i]t is a hallmark of the theory based on genuine fortuitousness that it does not admit physical variables. It is, therefore, of a novel kind that does not deal with things (objects in space), or measurements, and may be referred to as the theory of no things. (p. 410)

Such approaches to the interpretation of quantum mechanics are to be contrasted with realist views like Bohmian mechanics [11]. Here particles are taken to exist and they follow deterministic trajectories. For illustration, 
consider a set of $N$ nonrelativistic spin-zero particles interacting through a potential. The system's wave function is a solution of the Schrödinger equation. We can write it in polar form as

$$
\Psi\left(\mathbf{r}_{1}, \ldots, \mathbf{r}_{N}, t\right)=\rho \exp (i S / \hbar) .
$$

The equation of motion of particle $i$ is given by

$$
m_{i} \mathbf{v}_{i}=\nabla_{i} S .
$$

In general, $S$ is a nonadditive and nonseparable function of all particle coordinates $\mathbf{r}_{i}$. This can be shown to account for nonlocal effects like long-distance correlations.

In Bohmian mechanics, the statistical properties of quantum mechanics arise from an incomplete knowledge of the system's initial conditions. Indeed the particles' initial positions, although well-defined, are "hidden" to any observer. What is known is their statistical distribution, taken to be proportional to the absolute square of the wave function. It can be shown that Bohmian mechanics exactly reproduces the statistical results of quantum mechanics. In particular [22], interference fringes in Young's two-slit setup follow directly from (5).

If Bohmian mechanics exactly reproduces the statistical results of quantum mechanics, aren't the trajectories superfluous, and shouldn't they be discarded? The analogy has been made between such trajectories and the concept of the ether prevalent at the turn of the twentieth century [23, 24]. H. A. Lorentz and his contemporaries viewed electromagnetic phenomena as taking place in a hypothetical medium called the ether. From this, Lorentz developed a description of electromagnetism in moving reference frames, and he found that the motion is undetectable. Following Einstein's formulation of the electrodynamics of moving bodies, the ether was recognized as playing no role, and was henceforth discarded. So should it be, according to most proponents of the epistemic view of quantum states, with interpretations of quantum mechanics that posit observer-independent elements of reality like Bohmian trajectories. They predict no empirical differences with the Hilbert space formalism, and therefore should be discarded.

\section{Two explanatory functions}

To examine how appropriate are the epistemic and related views of quantum mechanics, it is important to properly understand the explanatory role of 
quantum mechanics as a physical theory. Although all measurements are made by means of macroscopic apparatus, quantum mechanics is used, as an explanatory theory, in two different ways: it is meant to explain (i) nonclassical correlations between macroscopic objects and (ii) the small-scale structure of macroscopic objects. That these two functions are distinct is best shown by contrasting the world in which we live with a hypothetical, closely related one.

Roughly speaking, the hypothetical world is defined so that (a) for all practical purposes, all macroscopic experiments give results that coincide with what we find in the real world, and (b) its microscopic structure, if applicable, is different from the one of the real world. Let us spell this out in more detail.

In the hypothetical world large scale objects, i.e. objects much larger than atomic sizes, behave just like large scale objects in the real world. The trajectories of baseballs and airplanes can be computed accurately by means of classical mechanics with the use of a uniform downward force, air friction, and an appropriate propelling force. Waveguides and antennas obey Maxwell's equations. Steam engines and heat pumps work according to the laws of classical thermodynamics. The motion of planets, comets, and asteroids is well described by Newton's laws of gravitation and of motion, slightly corrected by the equations of general relativity.

Close to atomic scales, however, these laws may no longer hold. Except for one restriction soon to be spelled out, I shall not be specific about the changes that macroscopic laws may or may not undergo in the microscopic realm. Matter, for instance, could either be continuous down to the smallest scales, or made of a small number of constituent particles like our atoms. The laws of particles and fields could be the same at all scales, or else they could undergo significant changes as we probed smaller and smaller distances.

In the hypothetical world one can perform experiments with pieces of equipment like Young's two-slit setup, Stern-Gerlach devices, or MachZehnder interferometers. Let us focus on the Young type experiment. It makes use of two macroscopic objects which we label $E$ and $D$. These symbols could stand for "emittor" and "detector" if it were not that, as we shall see, they may not emit or detect anything. At any rate, $E$ and $D$ both have on and off states and work in the following way. Whenever $D$ is suitably oriented with respect to $E$ (say, roughly along the $x$ axis) and both are in the on state, $D$ clicks in a more or less random way. The average time interval between clicks depends on the distance $r$ between $D$ and $E$, and 


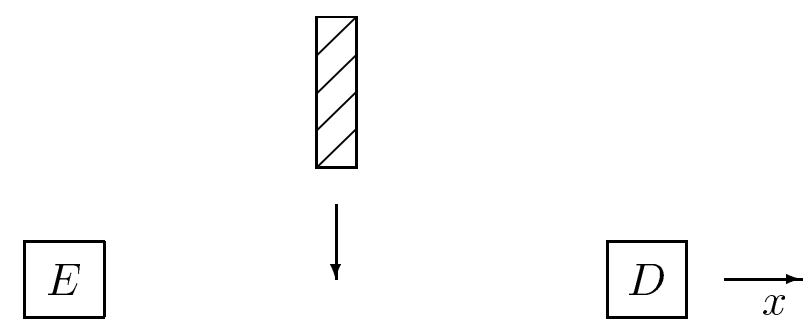

Figure 1: Shielding material prevents $D$ from clicking

falls roughly as $1 / r^{2}$. The clicking stops if, as shown in Fig. 1, a shield of a suitable material is placed perpendicularly to the $x$ axis, between $D$ and $E$.

If holes are pierced through the shield, however, the clicking resumes. In particular, with two small holes of appropriate size and separation, differences in the clicking rate are observed for small transverse displacements of $D$ behind the shield. A plot of the clicking rate against $D$ 's transverse coordinate displays maxima and minima just as in a wave interference pattern. No such maxima and minima are observed, however, if just one hole is open or if both holes are open alternately.

At this stage everything happens as if $E$ emitted some kind of particles and $D$ detected them, and the particles behaved according to the rules of quantum mechanics. Nevertheless, we shall nor commit ourselves to the existence or nonexistence of these particles, except on one count. Such particles, if they exist, are not in any way related to hypothetical constituents of the material making up $D, E$, or the shield, or of any macroscopic object whatsoever. Whatever the microscopic structure of macroscopic objects is, it has nothing to do with what is responsible for the correlations between $D$ and E.

In a similar way, we can perform in the hypothetical world experiments with Stern-Gerlach devices, Mach-Zehnder interferometers, or other setups used in the typical quantum-mechanical investigations carried out in the real world. Correlations are observed between initial states of "emittors" and final states of "detectors" which are unexplainable by classical mechanics but follow the rules of quantum mechanics. We assume again that, if these correlations have something to do with the emission and absorption of particles, these are in no way related to eventual microscopic constituents of the macroscopic devices. 
In the experiments just described that relate to the hypothetical world, quantum mechanics correctly predicts the correlations between $D$ and $E$ (or other "emittors" and "absorbers") when suitable experimental configurations are set up. In these situations, the theory can be interpreted in (at least) two broadly different ways. In the first one, the theory is understood as applying to genuine microscopic objects, emitted by $E$ and detected by $D$. Perhaps these objects follow Bohmian-like trajectories, or behave between $E$ and $D$ in some other way compatible with quantum mechanics. In the other interpretation, there are no microscopic objects whatsoever going from $E$ to $D$. There may be something like an action at a distance. At any rate the theory is in that case interpreted instrumentally, for the purpose of quantitatively accounting for correlations in the stochastic behavior of $E$ and $D$.

In the hypothetical world we are considering, I believe that both interpretations are logically consistent and adequate. Of course, each investigator can find more satisfaction in one interpretation than in the other. The epistemic view of quantum mechanics corresponds to the instrumentalist interpretation. It simply rejects the existence of microscopic objects that have no other use than the one of predicting observed correlations between macroscopic objects.

In the world in which we live, however, the situation is crucially different. The electrons, neutrons, photons, and other particles that diffract or interfere are the same that one appeals to in order to explain the structure of macroscopic objects. Denying their existence, as is done in the approach of genuine fortuitousness, dissolves such explanatory power. Denying that they have states, as is done in the epistemic view, leaves one to explain the state of a macroscopic object on the basis of entities that have no state.

\section{Interpreting quantum mechanics}

The epistemic and related views therefore fail to account for the second explanatory role of quantum mechanics. To reinforce this conclusion, it is instructive to investigate what it means to interpret a theory.

With most physical theories, interpretation is rather straightforward. But this should not blind us to the fact that even very familiar theories can in general be interpreted in more than one way. A simple example is classical mechanics. 
Classical mechanics is based on a well-defined mathematical structure. This consists of constants $m_{i}$, functions $\mathbf{r}_{i}(t)$, and vector fields $\mathbf{F}_{i}$ (understood as masses, positions, and forces), together with the system of secondorder differential equations $\mathbf{F}_{i}=m_{i} \mathbf{a}_{i}$. A specific realization of this structure consists in a system of ten point masses interacting through the $1 / r^{2}$ gravitational force. A hypothesis may then assert that the solar system corresponds to this realization, if the sun and nine planets are considered pointlike and all other objects neglected. Predictions made on the basis of this model correspond rather well with reality. But obviously the model can be made much more sophisticated, taking into account for instance the shape of the sun and planets, the planets' satellites, interplanetary matter, and so on.

Now what does the theory have to say about how a world of interacting masses is really like? It turns out that such a world can be viewed in (at least) two empirically equivalent but conceptually very different ways. The first one consists in asserting that the world is made only of small (or extended) masses that interact by instantaneous action at a distance. The second way asserts that the masses produce everywhere in space a gravitational field, which then locally exerts forces on the masses. These two ways constitute two different interpretations of the theory. Each one expresses a possible way of making the theory true (assuming empirical adequacy). Whether the world is such that masses instantaneously interact at a distance in a vacuum, or a genuine gravitational field is produced throughout space, the theory can be held as truly realized.

Similar remarks apply to classical electromagnetism. The mathematical equations can be interpreted as referring to charges and currents interacting locally through the mediation of electric and magnetic fields. Alternatively, they can be viewed as referring to charges and currents only, interacting by means of (delayed) action at a distance [25].

In this respect, quantum mechanics seems different from all other physical theories. There appears to be no straightforward way to visualize, so to speak, the behavior of microscopic objects. This was vividly pointed out by Feynman [26, p. 129] who, after a discussion of Young's two-slit experiment with electrons, concluded that "it is safe to say that no one understands quantum mechanics. [...] Nobody knows how it can be like that." But the process of interpreting quantum mechanics lies precisely in taking up Feynman's challenge. It is to answer the question, "How can the world be for quantum mechanics to be true?"

If we adopt this point of view (known as the semantic view of theo- 
ries [27, 28]), we can understand the function of Bohmian trajectories or, for that matter, of other interpretative schemes of quantum mechanics. Each provides us with one clear way that the microscopic objects can behave so as to reproduce the quantum-mechanical rules and, therefore, the observable behavior of macroscopic objects. It is true that, just like the ether in special relativity, they don't lead to specific empirical consequences. But although they could be dispensed with in the hypothetical world of Sec. 3, they cannot in the real world unless, just like the ether was eventually replaced by the free-standing electromagnetic field, they are replaced by something that can account for the structure of macroscopic objects.

In all physical theories other than quantum mechanics, there are straightforward and credible answers to the question raised above, of "How can the world be for the theory to be true?". In quantum mechanics there are a number of answers, for instance Bohmian trajectories, multiple worlds, modal approaches, etc. None is straightforward, and none gains universal credibility. Should we then adopt the attitude of the epistemic or related views, which decide not to answer the question? I believe that, from a foundational point of view, this is not tenable. For how can we believe in a theory, if we are not prepared to believe in any of the ways it can be true, or worse, if we do not know any way that it can be true?

\section{Conclusion}

The epistemic view of quantum mechanics is an attempt to solve or attenuate the foundational problems of the theory. We have seen that it would succeed if quantum mechanics were used only to explain nonclassical correlations between macroscopic objects. But it is also used to explain the microscopic structure of macroscopic objects. Interpreting the theory means finding ways that it can be intelligible. A number of proposals go a long way towards this, but much work remains to be done to make some of them sufficiently clear and precise.

\section{Acknowledgment}

This work was supported by the Natural Sciences and Engineering Research Council of Canada. 


\section{References}

[1] A. Einstein, B. Podolsky, and N. Rosen, "Can quantum-mechanical description of physical reality be considered complete?" Physical Review, 47: 777-780, May 1935.

[2] C. H. Bennett and G. Brassard, "Quantum cryptography: public key distribution and coin tossing," Proceedings of the IEEE International Conference on Computers, Systems and Signal Processing, New York: IEEE, 1984, pp. 175-179.

[3] P. W. Shor, "Algorithms for quantum computation: discrete logarithms and factoring," Proceedings of the $35^{\text {th }}$ Annual Symposium on Foundations of Computer Science, S. Goldwasser, Ed. Los Alamitos, CA: IEEE, 1994, pp. 124-134.

[4] G. 't Hooft, "Quantum gravity as a dissipative deterministic system," Classical and Quantum Gravity, 16: 3263-3279, October 1999.

[5] A. J. Leggett, "Testing the limits of quantum mechanics: motivation, state of play, prospects," Journal of Physics: Condensed Matter, 14: R415-R451, April 2002.

[6] C. Rovelli, "Relational quantum mechanics," International Journal of Theoretical Physics, 35: 1637-1678, August 1996.

[7] C. A. Fuchs and A. Peres, "Quantum theory needs no 'interpretation'," Physics Today, 53: 70-71, March 2000.

[8] C. A. Fuchs, "Quantum mechanics as quantum information (and only a little more)," in Quantum Theory: Reconsideration of Foundations, A. Khrennikov, Ed. Växjö: Växjö U. Press, 2002, pp. 463-543. Also available as quant-ph/0205039.

[9] P. E. Vermaas, A Philosopher's Understanding of Quantum Mechanics. Possibilities and Impossibilities of a Modal Interpretation, Cambridge: Cambridge U. Press, 1999.

[10] H. Everett III, “ 'Relative state' formulation of quantum mechanics," Reviews of Modern Physics, 29: 454-462, July 1957. 
[11] D. Bohm, "A suggested interpretation of the quantum theory in terms of 'hidden' variables (I and II)," Physical Review, 85: 166-193, January 1952.

[12] L. Marchildon, "Why should we interpret quantum mechanics?" Foundations of Physics, 34: 1453-1466, October 2004.

[13] L. Marchildon, "Bohmian trajectories and the ether: Where does the analogy fail?" Studies in History and Philosophy of Modern Physics, 37: 263-274, June 2006.

[14] L. Marchildon, "The epistemic view of quantum states and the ether," Canadian Journal of Physics, 84: 523-529, January 2006.

[15] L. E. Ballentine, "The statistical interpretation of quantum mechanics," Reviews of Modern Physics, 42: 358-381, October 1970.

[16] W. Heisenberg, Physics and Philosophy. The Revolution in Modern Science, New York: Harper, 1958.

[17] J. von Neumann, Mathematical Foundations of Quantum Mechanics, Princeton: Princeton U. Press, 1955.

[18] R. Peierls, "In defence of 'measurement'," Physics World, 4: 19-20, January 1991.

[19] I. Bloch, "Some relativistic oddities in the quantum theory of observation," Physical Review, 156: 1377-1384, April 1967.

[20] O. Ulfbeck and A. Bohr, "Genuine fortuitousness. Where did that click come from?" Foundations of Physics, 31: 757-774, May 2001.

[21] A. Bohr, B. R. Mottelson, and O. Ulfbeck, "The principle underlying quantum mechanics," Foundations of Physics, 34: 405-417, March 2004.

[22] C. Philippidis, C. Dewdney, and B. J. Hiley, "Quantum interference and the quantum potential," Il Nuovo Cimento, 52B: pp. 15-28, July 1979.

[23] J. Bub, "Why the quantum?" Studies in History and Philosophy of Modern Physics, 35: 241-266, June 2004. 
[24] J. Bub, "Quantum mechanics is about quantum information," Foundations of Physics, 35: 541-560, April 2005.

[25] J. A. Wheeler and R. P. Feynman, "Classical electrodynamics in terms of direct interparticle action," Reviews of Modern Physics, 21: 425-433, July 1949.

[26] R. P. Feynman, The Character of Physical Law, Cambridge, MA: MIT Press, 1967.

[27] R. N. Giere, Explaining Science. A Cognitive Approach, Chicago: U. of Chicago Press, 1988.

[28] F. Suppe, The Semantic Conception of Theories and Scientific Realism, Urbana: U. of Illinois Press, 1989. 